\title{
Delayed Blue Fluorescence via Upper-Triplet State Crossing from C-C Bonded Donor-Acceptor Charge Transfer Molecules with Azatriangulene Cores
}

Jonathan S. Ward, ${ }^{\dagger}{ }^{\dagger}$ Nadzeya A. Kukhta, ${ }^{\dagger}$ Paloma L. dos Santos, ${ }^{\ddagger}$ Daniel G. Congrave, ${ }^{\dagger}$ Andrei S. Batsanov, ${ }^{\dagger}$ Andrew P. Monkman, ${ }^{*}{ }^{\ddagger}$ and Martin R. Bryce ${ }^{* \dagger}+$ (1)

${ }^{\dagger}$ Department of Chemistry, Durham University, Durham DH1 3LE, U.K.

${ }^{\ddagger}$ Department of Physics, Durham University, Durham DH1 3LE, U.K.

\section{Supporting Information}

ABSTRACT: We report the synthesis and structural and photophysical characterization of two series of molecules with functionalized azatriangulene electron donor cores and three pendant electron acceptor units. The presented donor and acceptor units are joined by $\mathrm{C}-\mathrm{C}$ bonds, instead of the usual $\mathrm{C}$-heteroatom bonds often found in thermally activated delayed fluorescence (TADF) emitters. The effects of the donor-acceptor strength and donor-acceptor dihedral angle on the emission properties are assessed. The data establish that the singlet-triplet energy gap is $>0.3 \mathrm{eV}$ and that delayed emission is present in only specific host matrices, irrespective of host polarity. Specific host behavior is atypical of many TADF materials, and we suggest the delayed emission in this work does not occur by a conventional vibronically coupled TADF mechanism, as the $\Delta E_{\mathrm{ST}}$ value is too large. Detailed photophysical analysis and supporting density functional theory calculations suggest that some presented azatriangulene molecules emit via an upper-triplet state crossing mechanism. This work highlights that several different mechanisms can be responsible for delayed emission, often with highly similar photophysics. Detailed photophysical analysis is required to establish which delayed emission mechanism is occurring. Our results also highlight a clear future direction toward vibronically coupled $\mathrm{C}-\mathrm{C}$ bonded TADF materials.

\section{INTRODUCTION}

Conjugated aromatic organic molecules have been utilized innovatively in organic light-emitting diodes (OLEDs) as emitting materials and host materials, ${ }^{1-6}$ organic photovoltaics (OPVs) ${ }^{7-10}$ organic field effect transistors (OFETs), ${ }^{11,12}$ sensing, ${ }^{13-15}$ and bioelectronics. ${ }^{16,17}$ Minimization of nonradiative decay and channelling excited states through desired pathways can result in materials with emission quantum yields of $100 \%{ }^{18,19}$ A key challenge at present is to fully utilize triplet excited states that constitute $75 \%$ of the excitons generated under electrical operation. This issue has been tackled effectively with heavy metal complexes containing metals such as iridium $^{20-22}$ and platinum, ${ }^{23-25}$ where spin orbit coupling (SOC) facilitates direct emission from the triplet state as phosphorescence. There is currently great interest in developing all-organic systems that can harvest triplet excitons for emission. Organic molecules with conjugational separation between electron donor (D) and acceptor (A) units can result in a system in which a local triplet state $\left({ }^{3} \mathrm{LE}\right)$, a triplet charge transfer state $\left({ }^{3} \mathrm{CT}\right)$, and a singlet charge transfer state $\left({ }^{1} \mathrm{CT}\right)$ are close in energy. In this situation, harvesting of triplet states into singlet states can occur by reverse intersystem crossing (rISC). This process has previously been named as E-type delayed fluorescence but has also been called thermally activated delayed fluorescence (TADF). ${ }^{26,27}$ The ${ }^{3} \mathrm{CT}$ state participates in the rISC process through vibronic mixing of states. ${ }^{28-31}$ More recently, materials that harvest triplets by rISC from higher triplet states $\left(\mathrm{T}_{n}\right)$ have also been identified. ${ }^{32}$ These have been termed upper-triplet or hot exciton-harvesting emitters and often show photophysics similar to that of vibronically coupled TADF. ${ }^{33}$

TADF was identified in purely organic molecules in $1961^{34}$ but was not utilized in OLEDs with high efficiencies until 2012 by Adachi and co-workers. ${ }^{26}$ Subsequently, a large number of TADF molecules have been synthesized, with many exploited in OLEDs to achieve high efficiency. ${ }^{19}$ Obtaining satisfactory materials stability is still a significant challenge within the OLED field. This is an issue particularly for OLED devices emitting in the blue region, due to the high energy of the excited states in devices. $^{35}$ The instability of high-efficiency blue OLED materials

Special Issue: Jean-Luc Bredas Festschrift

Received: March 25, 2019

Revised: $\quad$ May 9, 2019

Published: May 22, 2019 
is an ongoing issue that research during the past 30 years has not yet solved. Stability at the operational voltages required for blue emission is mainly an issue in the emissive layer. Molecules in the emissive layer must be stable as cations, as anions, and in the neutral excited state. ${ }^{36}$ Density functional theory (DFT) studies by Song and Lee demonstrate that the bond dissociation energies of selected bonds in commonly used ambipolar OLED emitters/hosts are often low in one charged form. ${ }^{36}$ Crucially, twisted $\mathrm{sp}^{2} \mathrm{C}$-heteroatom bonds are identified as weak bonds within various sytems, ${ }^{36}$ and they are commonly used in TADF materials to achieve spatial separation of HOMO and LUMO. On the basis of the data of Song and Lee, there is a limited choice of functional groups available to prepare blue TADF emitters that have the potential to be stable under electrical device operation. Therefore, a molecular design strategy toward allorganic vibronically coupled TADF materials with good stability is of fundamental importance to the field. As a result, herein are reported the synthesis and characterization of six new donoracceptor molecules $\mathbf{1 a}-\mathbf{c}$ and $\mathbf{2 a - c}$ using an azatriangulene donor core with varying aryl acceptors. $\mathrm{C}-\mathrm{C}$ bonding between the donor and acceptor is a pivotal part of the new molecular design. The main motivation was to assess if vibronic-couplingtype TADF could be developed with a $\mathrm{C}-\mathrm{C}$ bond as the donoracceptor bridge. All molecules are highly emissive with PLQY values ranging from $43 \%$ to $67 \%$ in drop-cast thin films. A detailed analysis of $1 a-c$ and $2 a-c$ reveals that $1 c$ and $2 c$ show some delayed fluorescence via upper-triplet state crossing in the mCBP host. The presented results also reveal key future steps that are required to obtain vibronically coupled TADF from a $\mathrm{C}-\mathrm{C}$ bonded donor-acceptor molecule.

\section{RESULTS AND DISCUSSION}

Synthesis. The design strategy for the new molecular series considered the reported stability of functional groups. ${ }^{36}$ The rationale is summarized in Figure 1.

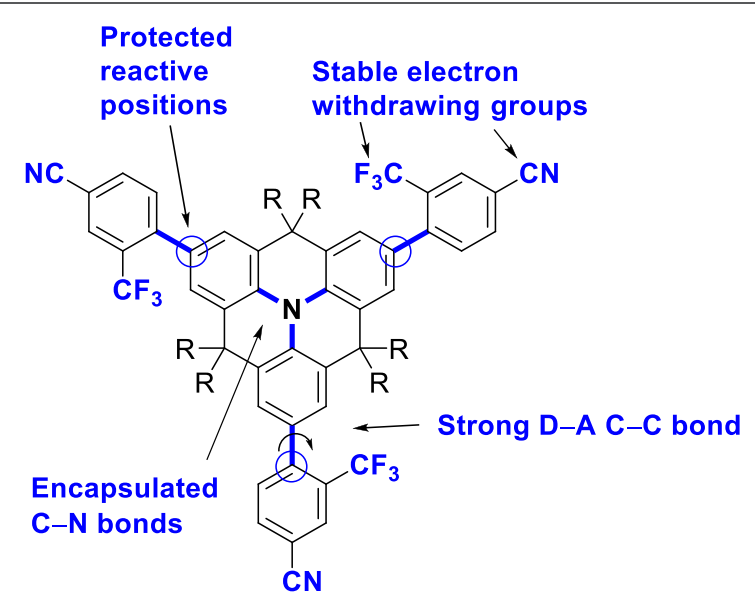

Figure 1. Design strategy for creating new blue emissive materials. Representative molecules $\mathbf{1 b}(\mathrm{R}=\mathrm{Me})$ and $\mathbf{2 b}(\mathrm{R}=\mathrm{Ph})$ are shown.

On the basis of a report by Song and Lee, ${ }^{36}$ potential sites of electrochemical instability are blocked, the $\mathrm{sp}^{2} \mathrm{C}$-heteroatom bonds are encapsulated to protect the weakest bonds in the system, and the $\mathrm{D}-\mathrm{A}$ units are linked by $\mathrm{C}-\mathrm{C}$ bonds. Literature reports also highlight $\mathrm{CF}_{3}$ and $\mathrm{CN}$ as stable functional groups. $^{37,38}$ On the basis of the strategy highlighted in Figure 1 , the newly prepared molecules are shown in Figure 2.
The aim of the two series of molecules was to assess the effect of the varying donor-acceptor strength and D-A dihedral angle on the emission properties and to assess if the $\mathrm{D}-\mathrm{A}$ dihedral angle utilized would result in vibronically coupled TADF. It was also of interest to assess how varying donor substituents would affect the photophysical properties. Literature X-ray data report that the central donor unit of $1 \mathbf{a}-\mathbf{c}$ is planar, ${ }^{6}$ whereas the previously reported $p$-tolyl-substituted core (similar to the core of $2 a-c)$ is folded into a shallow "bowl" shape. ${ }^{39}$ Subtle folding affects were expected to alter the emission properties of the two series of molecules. The structures and purity of the new molecules were determined by characterization with ${ }^{1} \mathrm{H},{ }^{13} \mathrm{C}$ $\left\{{ }^{1} \mathrm{H}\right\}$, and ${ }^{19} \mathrm{~F}$ nuclear magnetic resonance (NMR) spectroscopy, high-resolution mass spectrometry, and X-ray single-crystal analysis. The full experimental procedures for the synthesis and the characterization data of the molecules in Figure 2 are detailed in the sections S6 and S7 of the Supporting Information. The synthesis of molecule $2 \mathrm{c}$ is shown as a representative example in Scheme 1.

New hexaphenyl core 5 was synthesized in two steps by treatment of triester 3 with $\mathrm{PhLi}$ to give triol 4. The triol intermediate was closed by reflux in acid to give $\mathbf{5}$ in $70 \%$ yield over the two steps. Tribromination of $\mathbf{5}$ proceeded cleanly in $\mathrm{CHCl}_{3}$ with $\mathrm{Br}_{2}$ with only aqueous workup required for purification to give 6. A 3-fold Suzuki coupling of 6 with 2,4dicyanobenzeneboronic acid pinacol ester gave $2 \mathrm{c}$ in $19 \%$ yield. The yield for the $\mathbf{2 a}-\mathbf{c}$ series was generally lower than that for the $1 \mathbf{a}-\mathbf{c}$ series, and this was likely due to the insolubility of tribromo precursor 6. The steric effect of the six peripheral phenyl substituents could explain the low reactivity of 6 .

Solution Electrochemistry and Thermal Properties. The electrochemical properties of the new compounds were studied by cyclic voltammetry $(\mathrm{CV})$ in solution. The results are summarized in Figure 3 and Table S1.

The first oxidation processes of molecules $\mathbf{1 a}-\mathbf{c}$ and $\mathbf{2 a - c}$ were studied. The voltammograms in Figure 3 show that increasing the acceptor strength in $\mathbf{1 a}-\mathbf{c}$ results in a significant shift of the oxidation potential. Using a stronger acceptor makes oxidation of the central azatriangulene core more difficult. Interestingly, the diphenylmethylene-substituted azatriangulene cores in $\mathbf{2 a}-\mathbf{c}$ are harder to oxidize than any members of the $\mathbf{1 a -}$ c series. The "bowl shape" of the donor unit in $\mathbf{2 a}-\mathbf{c}$ weakens the ability of the central " $\mathrm{N}$ " to donate to the connected phenyl rings. Therefore, $\mathbf{2 a}$ is harder to oxidize than all members of the $\mathbf{1 a}-\mathbf{c}$ series, despite having only a single withdrawing group on each acceptor unit. The oxidation processes were assessed for chemical reversibility by cycling the oxidation waves three times (Figure S5a). Compound 2a shows an irreversible oxidation based on the shift of the oxidation potential to higher energies with each cycle. In contrast, the oxidations for $1 \mathrm{a}-\mathrm{c}$ are reversible and for $\mathbf{2 b}$ and $\mathbf{2 c}$ are quasi-reversible. The stronger acceptors on $\mathbf{2 b}$ and $\mathbf{2 c}$ prevent the degradation processes from occurring in solution compared to $2 \mathrm{a}$. All reduction processes for $1 \mathrm{a}-\mathrm{c}$ and $\mathbf{2 a}-\mathbf{c}$ are irreversible or are at potentials too high for detection within the solvent window. Irreversible reductions are unsurprising considering the wide HOMO-LUMO energy gaps and the low oxidation potentials of the materials. Through rational molecular design, it is now possible to control whether the oxidation processes are reversible in these $\mathrm{D}-\mathrm{A}_{3}$ systems.

Thermogravimetric analysis (TGA) was performed on 1a-c and $2 a-c$ for an assessment of thermal stability (Figure S5b). Clear differences in the thermal versus electrochemical stability are observed across the series. 1a shows high electrochemical 


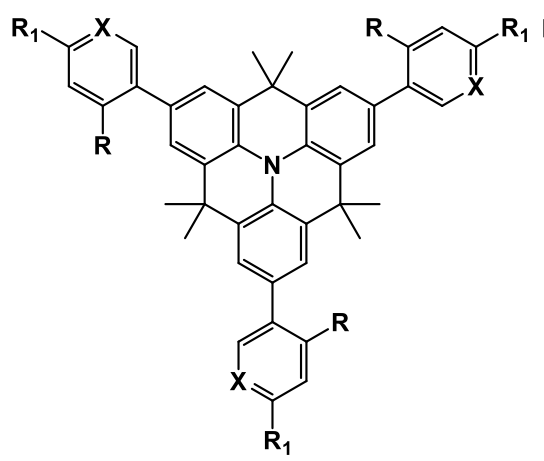

1a, $\mathrm{R}=\mathrm{CN}, \mathrm{R}_{1}=\mathrm{H}, \quad \mathrm{X}=\mathrm{CH}$

1b, $R=C_{3}, R_{1}=C N, X=C H$

1c, $R=C F_{3}, R_{1}=C F_{3}, X=N$<smiles>[R]c1ccc(-c2ccc3c(c2)C(c2ccccc2)(c2ccccc2)c2cc(-c4ccc(C)cc4[R])cc4c2Nc2c-3cc(-c3ccc([R7])cc3[R])cc2C4(c2ccccc2)c2ccccc2)c([R])c1</smiles>

2a, R = CN, $R_{1}=H$

$2 b, R=C F_{3}, R_{1}=C N$

2c, $R=C N, R_{1}=C N$

Figure 2. Structures of the new molecules synthesized in this study. Series $1 \mathbf{a}-\mathbf{c}$ has the dimethylmethylene-substituted azatriangulene core, while series $2 a-c$ has the diphenylmethylene functionality.

Scheme 1. Synthetic Route to Compound 2c from Triester 3

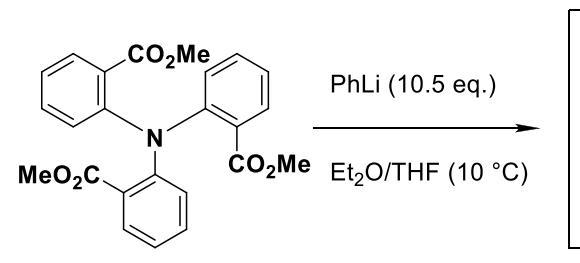

3

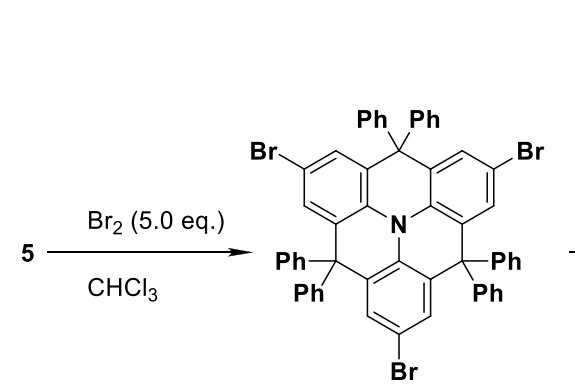

6, $91 \%$

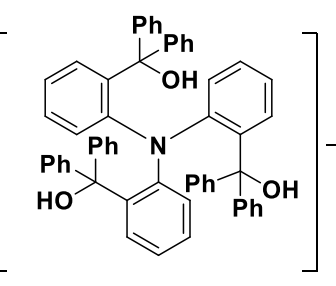

4

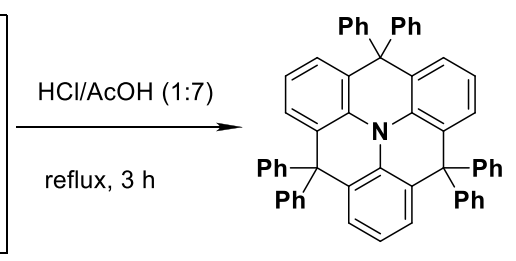

5, $70 \%$ from 3

$\operatorname{Pd}(\mathrm{OAc})_{2}(10 \mathrm{~mol} \%)$

SPhos (20 mol\%)

$\mathrm{Na}_{2} \mathrm{CO}_{3}$ (15 eq.)
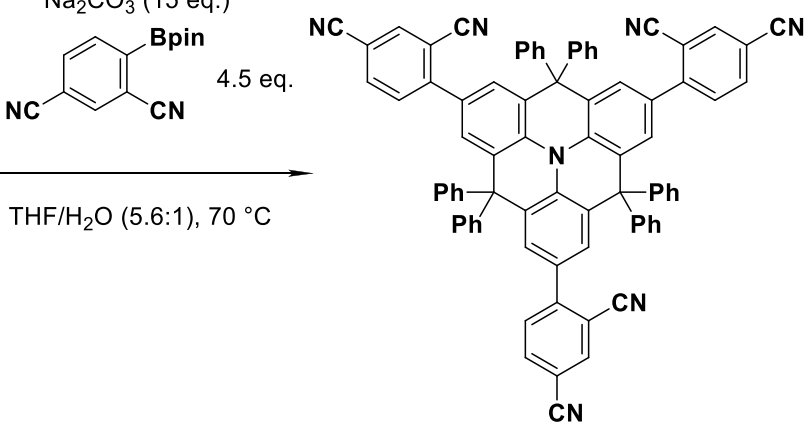

2c, $19 \%$
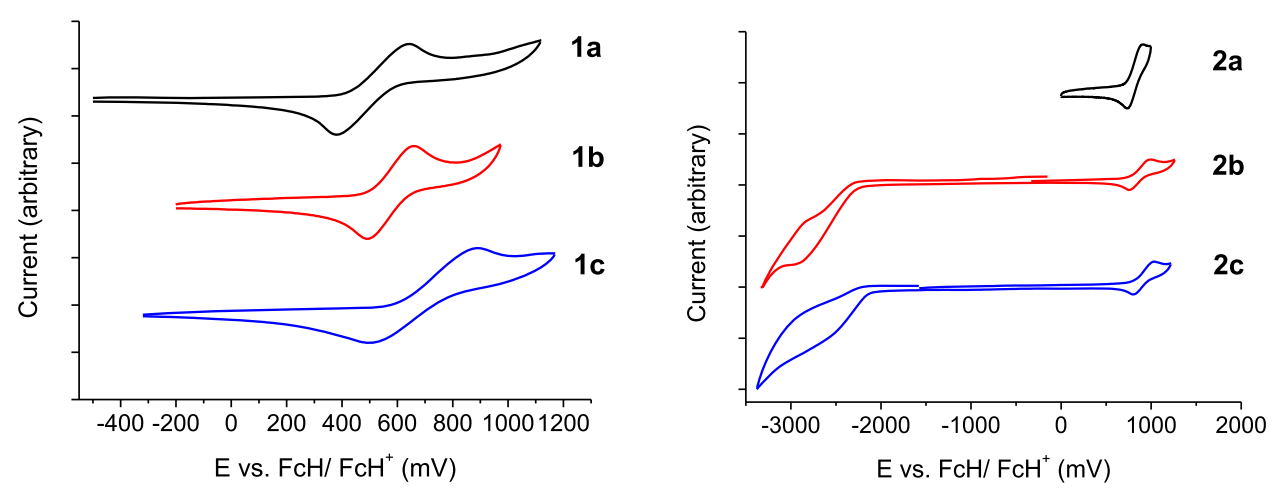

Figure 3. Cyclic voltammograms of $1 \mathrm{a}-\mathrm{c}$ and $\mathbf{2 a}-\mathrm{c}$ in $0.1 \mathrm{M} \mathrm{TBAPF}_{6}$ solution in dry tetrahydrofuran.

stability but is unexpectedly sensitive to temperature with 5\% mass loss at only $137^{\circ} \mathrm{C}$. $\mathbf{1 b}$ shows good thermal stability (349 ${ }^{\circ} \mathrm{C}$ ), but the pyridine unit within $1 \mathrm{c}$ appears to reduce stability $\left(253{ }^{\circ} \mathrm{C}\right)$. Notably, $2 \mathrm{a}-\mathrm{c}$ all show very good thermal stability with $5 \%$ mass loss values at $>400{ }^{\circ} \mathrm{C}$. The bisphenylmethylene substitutents in the new donor core unit clearly improve the 

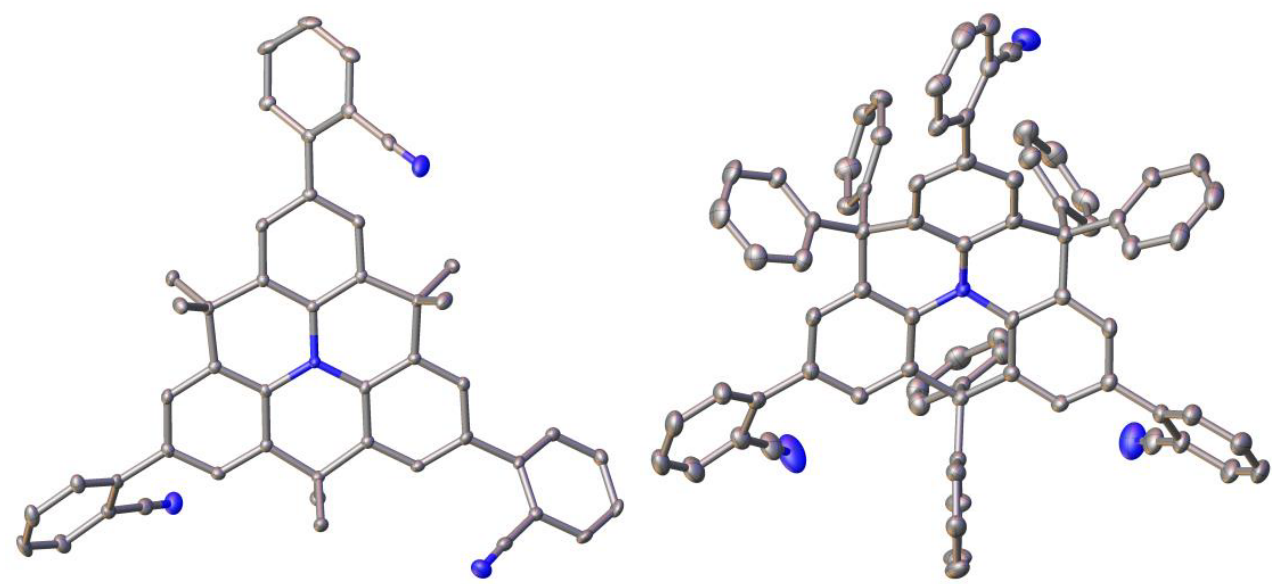

Figure 4. X-ray molecular structures of $1 \mathrm{a}$ in $1 \mathrm{a} \cdot \mathrm{CH}_{2} \mathrm{Cl}_{2}$ and 2a. Thermal ellipsoids are drawn at the $50 \%$ probability level. $\mathrm{H}$ atoms are omitted.

thermal stability of $2 a-c$ compared to that of the known donor core in $1 \mathrm{a}-\mathbf{c}$.

X-ray Crystallography. Molecular structures of $\mathbf{1 a}-\mathbf{c}$ and $2 \mathbf{a}-\mathbf{c}$ in the solids are shown in Figure 4 and Figure $54 a-d$. Selected geometrical parameters are listed in Table S2. The core in molecule $\mathbf{1 a}$ is nearly planar; in $\mathbf{1 b}$, it is twisted but the central $\mathrm{N}$ atom retains nearly planar-trigonal geometry (Figure S4c). In all other molecules, the core is "bowl-shaped" and the $\mathrm{N}$ atom is substantially pyramidal, displaced by $\sim 0.2 \AA$ from the plane of the three adjacent $\mathrm{C}$ atoms [C3 plane (see Table S2)]. The angles between the core arene rings $(\mathrm{Ar} / \mathrm{Ar})$ and between these arenes and the $\mathrm{C} 3$ plane $(\mathrm{C} 3 / \mathrm{Ar})$ are broadly similar in all structures except 1a. The twist angles between the acceptor arene rings and the adjacent donor vary widely, both between different compounds and among three independent molecules in the structure of $\mathbf{2 b}$.

In 1a, the acceptor units have their $o-\mathrm{CN}$ substituents on both sides of the core plane; the same is true for the $o-\mathrm{CF}_{3}$ groups in 1b. In $\mathbf{1 c}$ and $\mathbf{2 b}$, all $o-\mathrm{CF}_{3}$ groups are on the same (concave) side of the core. Remarkably, in the nonsolvated crystal of $2 a$, all $o$-CN groups are on the convex side, and in the DCM solvate of $\mathbf{2 a}$, on the concave side of the core (Figure S4c). In $\mathbf{2 c}$, two $o$-CN groups are on the concave side and one is on the convex side.

Photophysical Properties. To determine the nature of the excited states and photophysical processes occurring in the presented molecules, a detailed study of the photophysics was undertaken. The absorption spectra of $1 \mathbf{a}-\mathbf{c}$ and $2 \mathbf{a}-\mathbf{c}$ in toluene are shown in Figure 5.

The absorption spectra of $\mathbf{1 a}-\mathbf{c}$ and $\mathbf{2 a}-\mathbf{c}$ generally show two bands. Molecules $\mathbf{1 a}$ and $\mathbf{1} \mathbf{b}$ have an absorption band at $\approx 330$
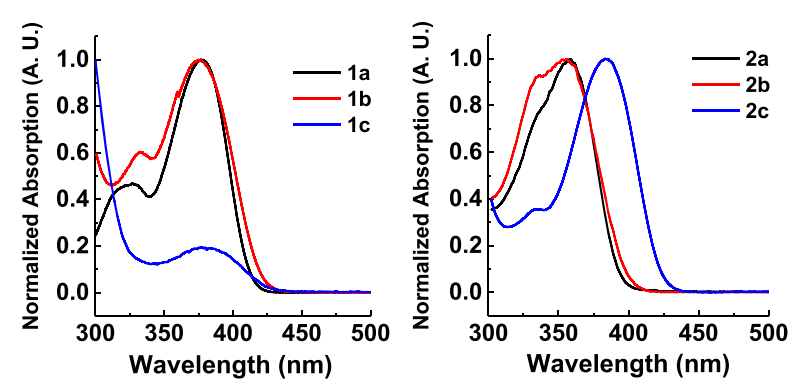

Figure 5. Normalized absorption spectra in toluene at ambient temperature for $\mathbf{1 a}-\mathbf{c}($ left $)$ and $\mathbf{2 a}-\mathbf{c}$ (right). Spectra are normalized at the highest intensity over the range of $300-500 \mathrm{~nm}$. $\mathrm{nm}$ and an additional lower-energy band at $375 \mathrm{~nm}$. There is little difference in the position of the lower-energy band in $\mathbf{1 a - c}$; however, the high-energy $\pi-\pi^{*}$ band of $1 \mathrm{c}$ is significantly blueshifted, presumably due to the strongly electron-withdrawing nature of the bis- $\mathrm{CF}_{3}$-pyridine acceptor. A similar absorption spectrum with a very high-energy band was observed in the literature for azatriangulene-triazine analogue HMAT-TRZ. ${ }^{40}$ $2 \mathrm{a}-\mathbf{c}$ have absorption profiles similar to those of $\mathbf{1 a}-\mathbf{c}$, but the low-energy band of $\mathbf{2 a}$ and $\mathbf{2 b}$ is significantly blue-shifted. It is suggested that the steric influence of the diphenylmethylene functionality of the donor in $\mathbf{2 a}-\mathbf{c}$ reduces the donor strength of the central core, giving rise to the observed blue-shift.

The steady state emission spectra of all molecules in solution state and solid state zeonex were obtained to assess initial photophysical behavior (Figure 6). The aliphatic zeonex polymer matrix has no electronic transitions in the visible region of the spectrum. Using molecules at a low concentration $(5-10 \%)$ in zeonex often simplifies emission measurements and prevents intermolecular interactions between $\pi$-systems. ${ }^{41}$

The steady state emission in solution (Figure 6a,b) shows that $\mathbf{1 a}-\mathbf{c}$ and $\mathbf{2 a - c}$ exhibit positive solvatochromism. There is a clear red-shift in the emission on switching from toluene to DCM as the solvent. The degree of positive solvatochromism increases in the order $\mathbf{a}<\mathbf{b}<\mathbf{c}$ for both series. Molecules $\mathbf{1} \mathbf{c}$ and $2 \mathrm{c}$ show the strongest solvatochromism due to the presence of stronger acceptor groups. As observed in the absorption spectra, the emission of $\mathbf{2} \mathbf{a}$ and $\mathbf{2 b}$ is at higher energy in both solvents compared to that of their corresponding analogues $\mathbf{1 a}$ and $\mathbf{1} \mathbf{b}$. This is more evident in the emission spectra of $\mathbf{2} \mathbf{b}$, where two bands are clearly observed in toluene, but the higher-energy band, which is predominantly from local emission, is lost in the DCM solvent. It appears that the donor in the $\mathbf{2 a}-\mathbf{c}$ series is capable of hindering charge transfer (CT), but use of a more polar solvent makes $\mathrm{CT}$ emission more favorable. Only a single broad band is observed in DCM for all molecules and is assigned as pure $\mathrm{CT}$ emission. For $\mathbf{1 a}, \mathbf{1} \mathbf{b}$, and $\mathbf{2} \mathbf{a}$ in toluene, the emission band is non-Gaussian, suggesting mixed local charge transfer character.

The emission of the studied molecules varies significantly in zeonex (Figure 6c,d) compared to the solution state (Figure $6 \mathrm{a}, \mathrm{b})$. In the solid state, two main factors are responsible for the change in the degree of charge transfer compared to solution. First, switching to the solid state restricts molecular movement and imparts packing effects, which can influence the degree of CT emission. Second, the solid state polarity also plays a 

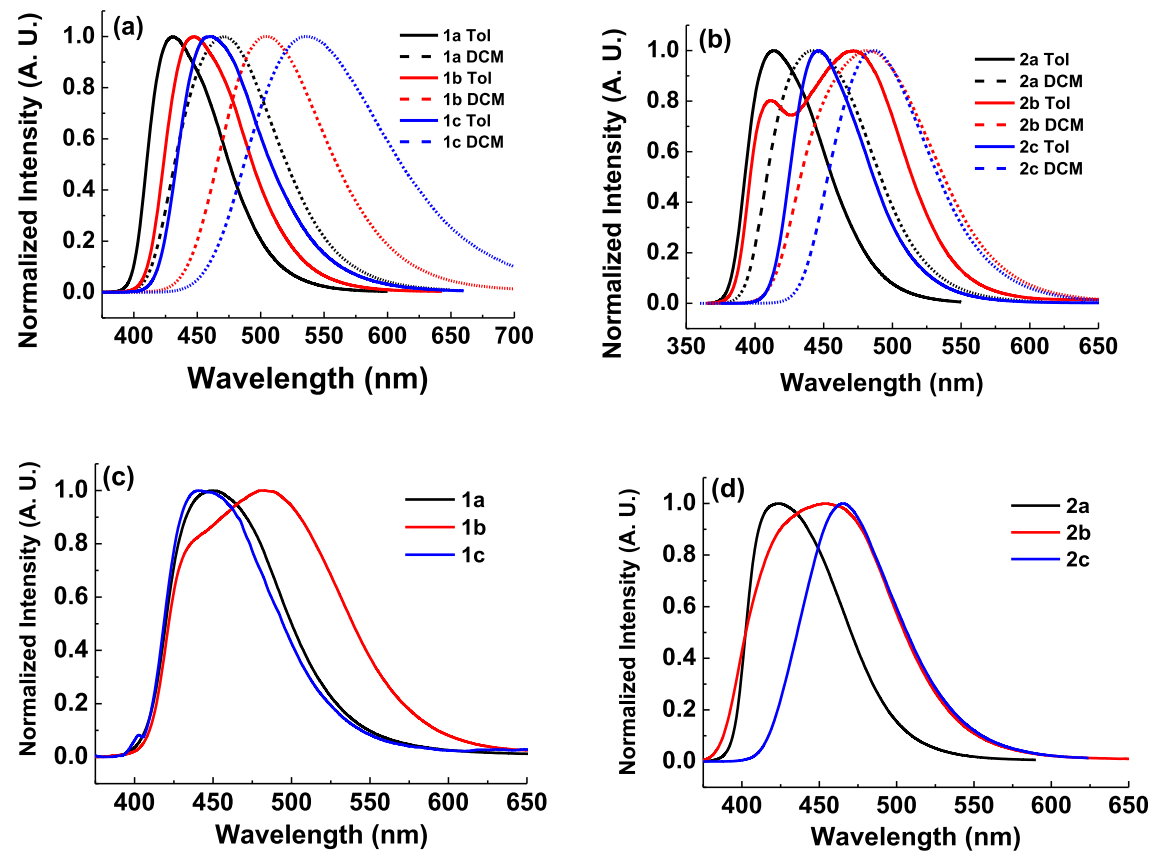

Figure 6. Steady state fluorescence spectra of molecules for (a) $\mathbf{1 a}-\mathbf{c}$ and (b) $\mathbf{2 a}-\mathbf{c}$ in toluene (Tol) and dichloromethane (DCM) at ambient temperature. Solid steady state photoluminescence of (c) $\mathbf{1 a}-\mathbf{c}$ and (d) $\mathbf{2 a}-\mathbf{c}$ in the zeonex matrix. The excitation wavelength for all measurements was $355 \mathrm{~nm}$.
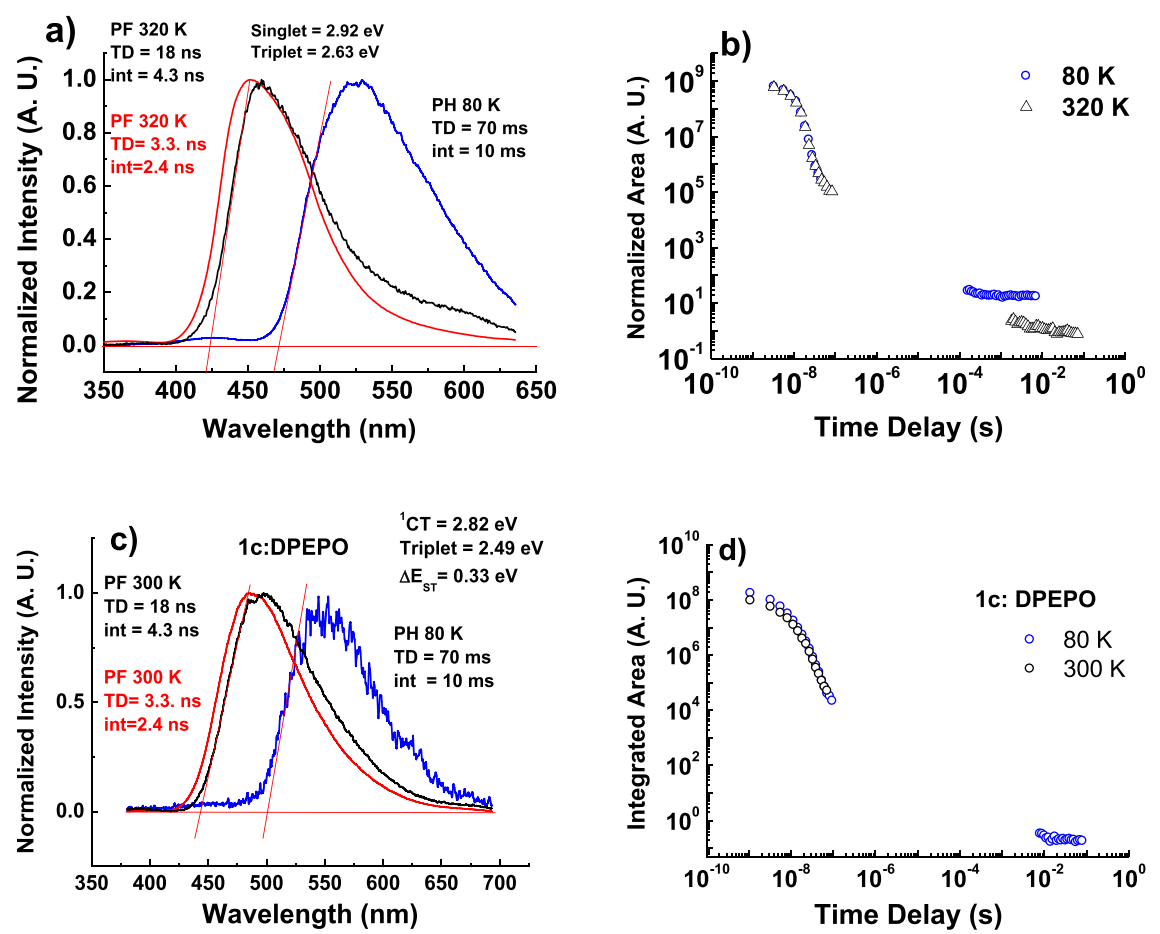

Figure 7. (a) Time-resolved emission spectra of 1a in zeonex showing prompt fluorescence (PF) and phosphorescence (PH) at a given temperature, time delay (TD), and integration time (int). (b) Time-resolved emission decay curve in zeonex matrix for $1 \mathrm{a}$ at 320 and $80 \mathrm{~K}$. The normalized area for each point represents all emission at all measured wavelengths by integration of the spectra collected at each delay time. Spectra in panel (a) were obtained from selected snapshots in time from the data shown in panel (b). The excitation wavelength was $355 \mathrm{~nm}$. (c) Time-resolved emission spectra of $10 \mathrm{wt} \% \mathbf{1 c}$ in polar DPEPO host. (d) Time-resolved decay curve in the zeonex matrix for $1 \mathrm{c}$ at $300 \mathrm{~K}$ in the DPEPO host. All measurements were performed in the absence of oxygen.

significant role in the degree of CT formation. The zeonex matrix is a nonpolar host with an aliphatic backbone and is therefore less polar than toluene or DCM. Both of these effects are responsible for the change in the degree of CT emission in this host. 1c gave the most red-shifted emission in solution, whereas the emission of $\mathbf{1 b}$ is the most red-shifted in the solid state. $1 \mathrm{c}$ in toluene has a single band and is still deep blue in color, suggesting dominant LE character. 1b shows dual emission suggesting a high content of the local excited (LE) state at a higher energy and emission from a state with a high CT 

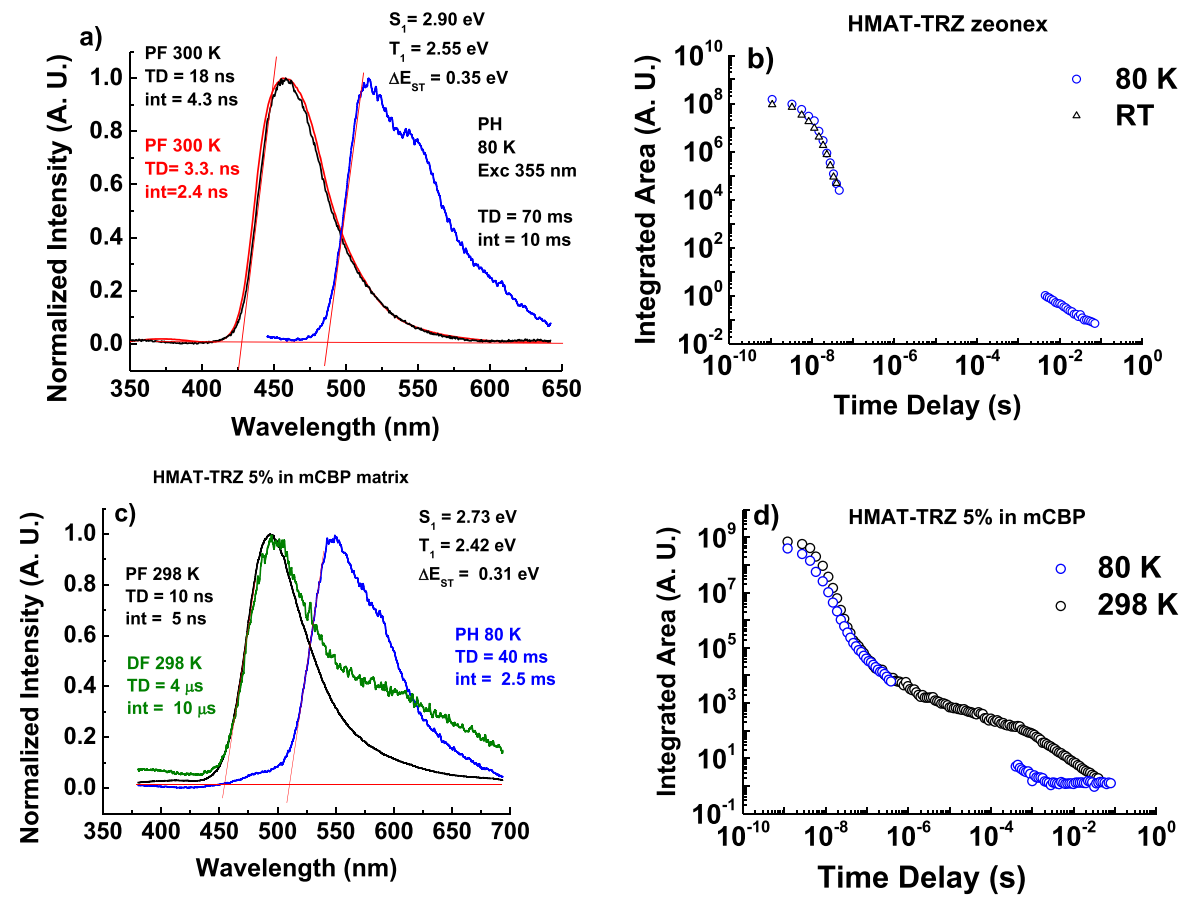

Figure 8. (a) Time-resolved emission spectra of HMAT-TRZ in zeonex matrix showing prompt fluorescence (PF) and phosphorescence (PH) at a given temperature, time delay (TD), and integration time (int). (b) Time-resolved emission decay curve zeonex matrix for HMAT-TRZ at 80 and 300 K. (c) Time-resolved emission spectra of 5 wt \% HMAT-TRZ in mCBP host showing prompt fluorescence (PF), delayed fluorescence (DF), and phosphorescence (PH) at a given temperature, time delay (TD), and integration time (int). (d) Time-resolved emission decay in mCBP for HMATTRZ at 80 and $298 \mathrm{~K}$. The excitation wavelength was $355 \mathrm{~nm}$. All measurements were performed in the absence of oxygen.

content at a lower energy. The dual emission of $\mathbf{1 b}$ in zeonex is reminiscent of the solution state spectra of $\mathbf{2 b}$, and the 2 trifluoromethyl-4-cyanobenzene acceptor is common to both $\mathbf{1 b}$ and $\mathbf{2 b}$. The acceptor in $\mathbf{1 b}$ and $\mathbf{2 c}$ appears to be on the borderline of being able to facilitate charge transfer in the nonpolar zeonex matrix. $\mathbf{1 b}$ and $\mathbf{2 c}$ have the highest CT character and require less host polarity to stabilize the CT state. Molecules $\mathbf{2 a}-\mathbf{c}$ in zeonex show a trend in emission color similar to that in solution. It is suggested that the steric effects of the donor bisphenylmethylene functionality in $2 a-c$ influence the geometry and CT strength in solution, while packing in the solid state is not playing a major role in contrast with $1 \mathbf{a}-\mathbf{c}$.

To assess if any of the molecules show delayed fluorescence (DF) in the zeonex matrix, time-dependent emission measurements were performed at 320 and $80 \mathrm{~K}$. Using time-resolved emission spectra with a given integration time, it is possible to determine the energy of singlet and triplet states, thus estimating the singlet-triplet energy gap $\left(\Delta E_{\mathrm{ST}}\right)$ of the emitters. Figure 7 shows the time-dependent emission data for $\mathbf{1 a}$ in the zeonex host.

To obtain the energy of the singlet CT state $\left({ }^{1} \mathrm{CT}\right)$, the prompt emission ( $<40 \mathrm{~ns}$ time range) at $320 \mathrm{~K}$ is used. To obtain the energy of the lowest-energy triplet state $\left(T_{1}\right)$, emission in the millisecond time range at $80 \mathrm{~K}$ was measured [phosphorescence $(\mathrm{PH})$. A long time delay of $70 \mathrm{~ms}$ is required to collect the phosphorescence to ensure that no other minor long-lived emission is collected. It is clear from the measurements in zeonex that the $\Delta E_{\mathrm{ST}}$ is $>0.3 \mathrm{eV}$ for $1 \mathrm{a}$ and that there is no delayed emission in the microsecond region. Molecule $\mathbf{1 b}$ was synthesized with the expectation that the $o-\mathrm{CF}_{3}$ substitution on the acceptor would improve the HOMO-LUMO separation and reduce $\Delta E_{\mathrm{ST}}$ due to an increased $\mathrm{D}-\mathrm{A}$ dihedral angle. $\mathbf{1 b}$ shows the desired red-shift of the ${ }^{1} \mathrm{CT}$ in solution (Figure 6a), but in zeonex, little shift is observed compared to that of $\mathbf{1 a}$ (Figure $6 \mathrm{c}$ and Figure $\mathrm{S} 2 \mathrm{a}$ ). A similar trend in $\Delta E_{\mathrm{ST}}$ for $\mathbf{1 a}-\mathrm{c}$ and $2 \mathrm{a}-\mathbf{c}$ regardless of the $\mathrm{D}-\mathrm{A}$ dihedral angle and acceptor strength was observed in zeonex. All $\Delta E_{\mathrm{ST}}$ values for $1 \mathrm{a}-\mathrm{c}$ and $2 \mathbf{a}-\mathbf{c}$ are $>0.3 \mathrm{eV}$, and no delayed emission is observed in the microsecond region, which would typically be expected for vibronically coupled TADF. It was postulated that a more polar solid state environment might red-shift the ${ }^{1} \mathrm{CT}$ energy and potentially narrow the $\Delta E_{\mathrm{ST}}$. Due to the strong bistrifluoromethylpyridine acceptor, $\mathbf{1 c}$ was therefore selected for further analysis in bis $\{2-[\operatorname{di}($ phenyl)phosphino $]$ phenyl $\}$ ether oxide (DPEPO) as the host material (Figure $7 \mathrm{c}, \mathrm{d}$ ).

Both the prompt fluorescence $(\mathrm{PF})$ emission and phosphorescence $(\mathrm{PH})$ of $\mathbf{1 c}$ in DPEPO were found to red-shift together, by $20-25 \mathrm{~nm}$ compared with zeonex measurements (Figure $\mathrm{S} 2 \mathrm{~b}$ ), with the $\Delta E_{\mathrm{ST}}$ remaining $>0.3 \mathrm{eV}$. In vibronically coupled TADF molecular designs such as the D-A-D molecule 2,7bis(9,9-dimethyl-acridin-10-yl)-9,9-dimethylthioxanthene-S,Sdioxide (DDMA- $\mathrm{TXO}_{2}$ ), the ${ }^{1} \mathrm{CT}$ energy can be tuned to a lower level in a more polar host, while the localized triplet state $\left({ }^{3} \mathrm{LE}\right)$ remains practically constant. ${ }^{42}$ The measurements of $\mathbf{1 c}$ strongly suggest that the lowest-energy triplet state is partially delocalized across both the donor and the acceptor units; i.e., it has a degree of CT character leading to a similar red-shift as observed for the ${ }^{1} \mathrm{CT}$ emission band. It appears, therefore, that the $\mathrm{C}-\mathrm{C}$ bond between the donor and acceptor units allows too much electronic communication between $\mathrm{D}$ and $\mathrm{A}$ units. This communication was not expected to be so extensive, even with a $\mathrm{D}-\mathrm{A}$ dihedral angle of $>60^{\circ}$ in the $o-\mathrm{CF}_{3}$-substituted acceptor compounds.

The data presented so far reveal that molecules $\mathbf{1 a}-\mathbf{c}$ and $\mathbf{2 a -}$ c do not show the conventional vibronic-coupling-type TADF or any kind of DF in the zeonex matrix. Our attention was 

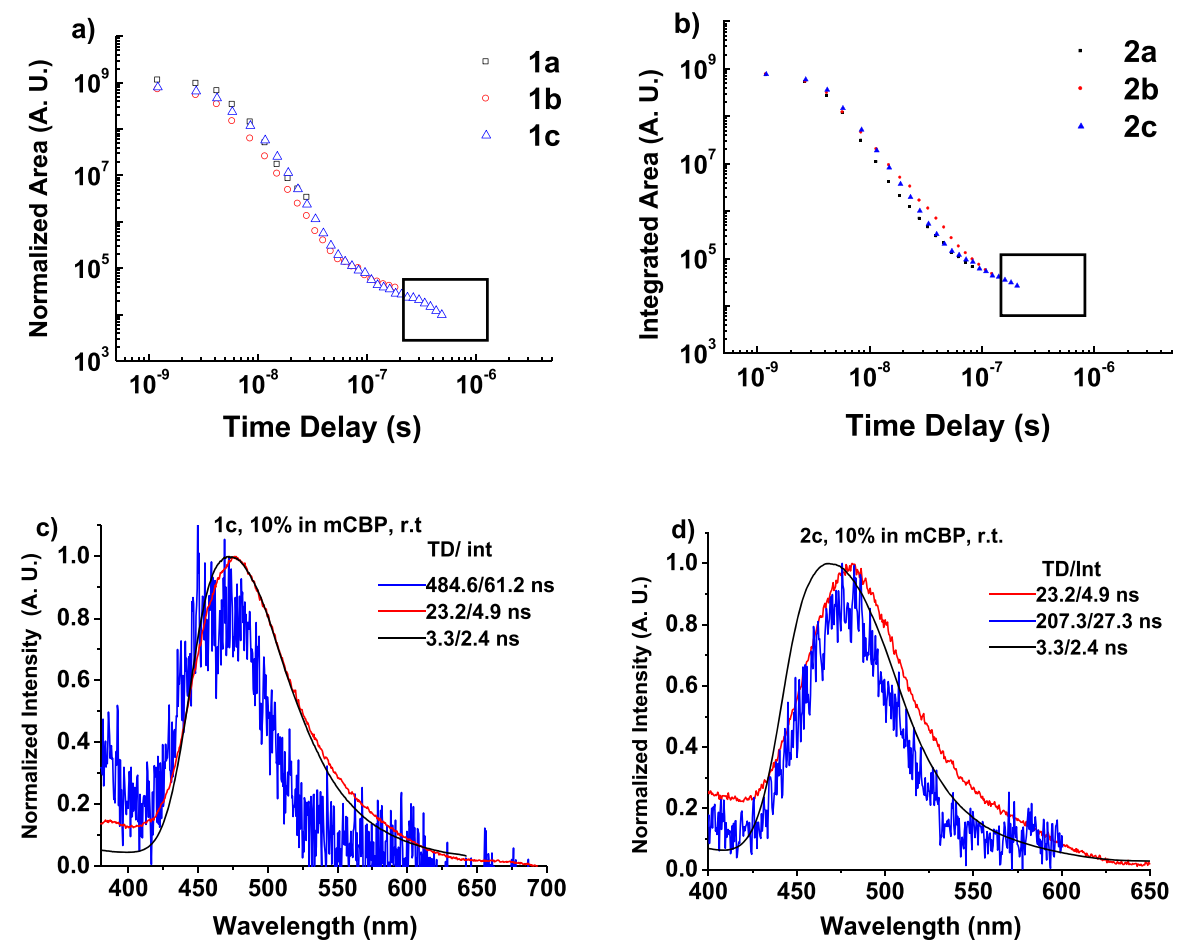

Figure 9. (a) Time-resolved emission decays of $10 \mathrm{wt} \% \mathbf{1 a}-\mathrm{c}$ in mCBP host. (b) Time-resolved emission decays of $10 \mathrm{wt} \% \mathbf{2 a}-\mathrm{c}$ in $\mathrm{mCBP}$ host. (c) Time-resolved emission spectra of $\mathbf{1} \mathbf{c}$ in $\mathrm{mCBP}$ host, showing prompt fluorescence (PF) and delayed fluorescence (DF) at a given time delay (TD) and integration time (int). (d) Time-resolved emission spectra of $2 \mathrm{c}$ in $\mathrm{mCBP}$ host showing prompt fluorescence (PF) and delayed fluoresence (PF) at a given temperature, time delay (TD), and integration time (int). The excitation wavelength was $355 \mathrm{~nm}$. All measurements were performed in the absence of oxygen at $298 \mathrm{~K}$. For panels (c) and (d), the blue trace indicates the last time frame where delayed fluorescence is clearly observed. In panels (a) and (b), the black boxes indicate the delayed emission in $\mathbf{1 c}$ and $\mathbf{2 c}$ that is not observed in $\mathbf{1 a}, \mathbf{1 b}, \mathbf{2 a}$, or $\mathbf{2 b}$.

therefore focused on the published analogous emitter hexamethylazatriangulene-triazine [HMAT-TRZ (its structure is shown in Figure S3a)], with the same core unit as $\mathbf{1 a}-\mathbf{c}$ and a single acceptor substituent, to gain greater insight into the processes occurring in $\mathbf{1 a}-\mathbf{c}$ and $\mathbf{2 a}-\mathbf{c}$. ${ }^{40}$ The previous study by Brédas and co-workers established that HMAT-TRZ exhibits some form of delayed emission despite a spectroscopically measured $\Delta E_{\mathrm{ST}}$ of $\approx 0.38 \mathrm{eV} \cdot{ }^{40} \mathbf{1 a}-\mathbf{c}$ and $\mathbf{2 a}-\mathbf{c}$ have reported $\Delta E_{\mathrm{ST}}$ values of $<0.38 \mathrm{eV}$ in most cases, and we were therefore interested in why HMAT-TRZ showed delayed emission but $\mathbf{1 a - c}$ and $\mathbf{2 a - c}$ did not. HMAT-TRZ was therefore resynthesized for comparison with the new materials 1a-c and $2 \mathbf{a}-\mathbf{c}$.

For initial comparison, emission measurements on HMATTRZ in zeonex were performed, as described for $\mathbf{1 a}-\mathbf{c}$ and $\mathbf{2 a}-\mathbf{c}$.

Apart from a slight red-shift and a lack of CT relaxation in the first $20 \mathrm{~ns}$, HMAT-TRZ shows photophysical properties highly similar to those of $\mathbf{1 a}-\mathbf{c}$ and $\mathbf{2 a}-\mathbf{c}$ in the zeonex matrix. The prompt fluorescence of HMAT-TRZ is observed in the nanosecond region followed by no delayed fluorescence (Figure $8 \mathrm{a}, \mathrm{b})$, with an $\Delta E_{\mathrm{ST}}$ value very comparable to those of $\mathbf{1 a}-\mathbf{c}$ and 2a-c. With a $\Delta E_{\mathrm{ST}}$ value in zeonex of $0.35 \mathrm{eV}$, this gap is too large to promote rISC between $\mathrm{T}_{1}$ and $\mathrm{S}_{1}$ in this host matrix. The published literature supplementary data establish that HMATTRZ shows delayed emission in the microsecond to millisecond region, but this is specifically dependent on the host matrix utilized. ${ }^{40}$ The reported host/guest blends of HMAT-TRZ that exhibit some form of delayed fluorescence show no trends with respect to host polarity. ${ }^{40}$ Previously reported DDMA-TXO2 shows vibronically coupled TADF in zeonex with clear prompt and delayed components, which is more efficient in the polar
DPEPO host due to host-guest tuning. ${ }^{42}$ HMAT-TRZ shows no TADF in the zeonex matrix (Figure 8) or in 1,3-bis $(N-$ carbazolyl)benzene ( $\mathrm{mCP})$ or the high-polarity DPEPO host. ${ }^{40}$ HMAT-TRZ does, however, show some form of weak delayed emission in intermediate-polarity hosts 3,3-di(9H-carbazol-9yl)biphenyl (mCBP) and $N, N^{\prime}$-bis $(N$-carbazolyl)-1,1'-biphenyl (CBP) (Figure S2h), and thus, time-resolved spectroscopy of HMAT-TRZ in mCBP was measured. It is very unusual that HMAT-TRZ (and indeed any vibronically coupled TADF emitter) requires such specific host materials to exhibit delayed emission. This is not routinely observed in efficient vibronically coupled TADF materials. ${ }^{19,42,43}$ Furthermore, the lifetime of the DF from HMAT-TRZ is much longer than that of typical TADF, reaching into the millisecond regime, which is way beyond the microsecond time scale common for vibronically coupled TADF. $^{44-47}$ It is not clear why $\mathrm{DPEPO}^{48}$ and $\mathrm{mCP}^{49}$ host would switch off delayed fluorescence from HMAT-TRZ, when DPEPO and $\mathrm{mCP}$ are deep-blue host materials with high triplet levels. Power dependence measurements performed in the CBP (Figure S2h) host show that the power dependence of delayed emission in the microsecond and millisecond region is close to unity. This result shows that the delayed emission is not due to a triplet-triplet annihilation mechanism (TTA). Thus, it is proposed that HMAT-TRZ exhibits delayed emission by a mechanism different from that of standard vibronically coupled TADF or TTA. Further studies and insight were required to identify an alternative mechanism, and this is discussed below.

HMAT-TRZ shows an essentially identical $\Delta E_{\mathrm{ST}}$ value $(\approx 0.3$ $\mathrm{eV}$ ) in zeonex, $\mathrm{mCBP}$, and polar diphenyl-4-triphenylsilylphenyl-phosphine oxide (TSPO1) hosts (Figure S2f), which is not typical of molecules that exhibit vibronically coupled 


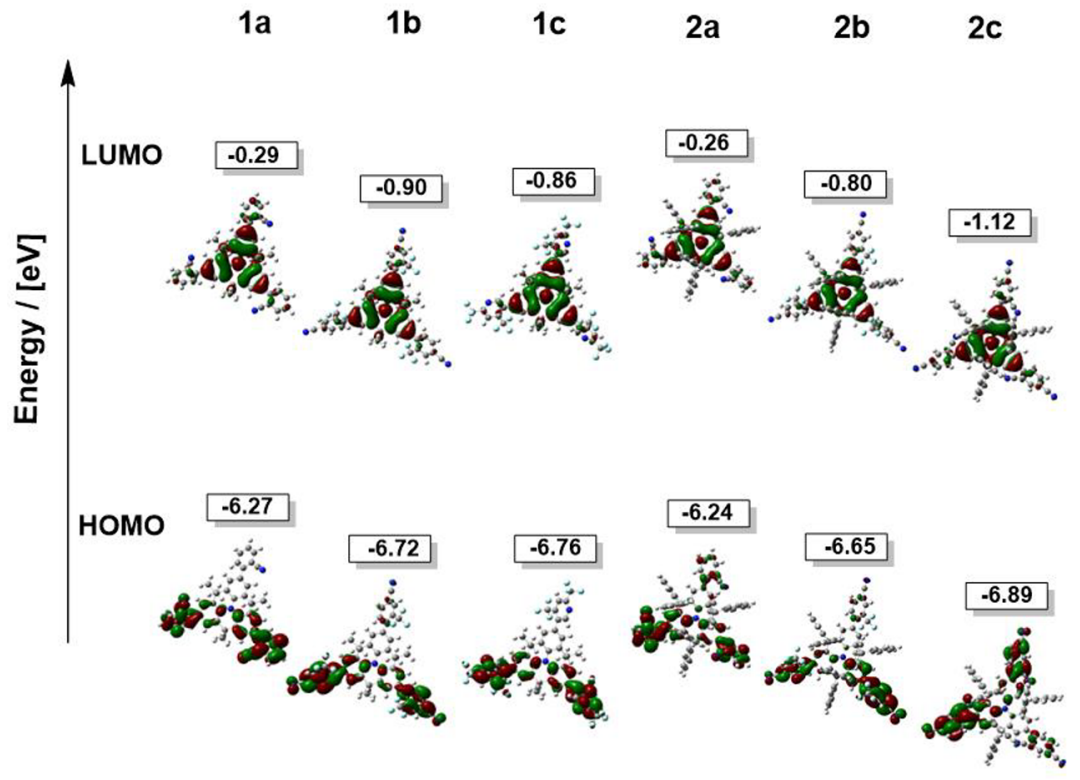

Figure 10. Calculated frontier orbitals of $\mathbf{1 a}-\mathbf{c}$ and $\mathbf{2 a}-\mathbf{c}[\mathrm{rCAM}-\mathrm{B} 3 \mathrm{LYP} / 6-31 \mathrm{G}(\mathrm{d})$ level].

TADF. $^{42,43,50}$ Changing the polarity of the host does not shift the singlet and triplet levels of HMAT-TRZ significantly closer in energy; both levels shift up or down in energy simultaneously. HMAT-TRZ shows some delayed emission in mCBP but not in zeonex, and therefore, comparable measurements in $\mathrm{mCBP}$ were also performed for $\mathbf{1 a}-\mathbf{c}$ and $\mathbf{2 a}-\mathbf{c}$ to assess if any delayed emission was present in these molecules (Figure 9).

Both series $\mathbf{1} \mathbf{a}-\mathbf{b}$ and $\mathbf{2} \mathbf{a}-\mathbf{b}$ do not show any delayed emission in the mCBP matrix, as was the case in zeonex. In contrast, both $1 \mathrm{c}$ and $2 \mathrm{c}$ show a relatively weak delayed emission component in $\mathrm{mCBP}$ in the range of 100-500 ns, which is not observed in zeonex matrix. In panels $\mathrm{c}$ and d of Figure 9, emission matching with prompt emission is clearly observed well beyond the initial $\mathrm{PF}$ exponential decay before $100 \mathrm{~ns}$. The delayed emission from 1c and $2 c$ is relatively weak but is clearly detectable with a sensitive iCCD camera and is not present in $\mathbf{1 a}, \mathbf{1} \mathbf{b}, \mathbf{2} \mathbf{a}$, or $\mathbf{2} \mathbf{b}$. Measurements on $\mathbf{1 c}$ in $\mathrm{mCBP}$ show that the delayed emission is dependent on temperature (Figure S2i), as previously demonstrated for HMAT-TRZ in the literature. ${ }^{40}$

The vibronic coupling mechanism for rISC relates specifically to the situation for low-exchange energy systems. In this case, ${ }^{1} \mathrm{CT}$ and ${ }^{3} \mathrm{CT}$ become degenerate such that no change in orbital angular moment can occur for a ${ }^{3} \mathrm{CT}$ to ${ }^{1} \mathrm{CT}$ transition; i.e., spin orbit coupling is forbidden. In this case, an energetically close third state is required, typically a local triplet state of D (or A). Vibrational coupling between ${ }^{3} \mathrm{CT}$ and ${ }^{3} \mathrm{LE}$ mixes these states such that SOC from ${ }^{3} \mathrm{CT}$ to ${ }^{1} \mathrm{CT}$ mediated by the ${ }^{3} \mathrm{LE}$ state can occur. This mechanism requires all three states, ${ }^{1} \mathrm{CT},{ }^{3} \mathrm{CT}$, and ${ }^{3} \mathrm{LE}$, to be close energetically. $k_{\mathrm{B}} T$ at $298 \mathrm{~K}$ is $26 \mathrm{meV}$, and for all molecules presented, the $\Delta E_{\mathrm{ST}}$ is generally $>300 \mathrm{meV}$, an order of magnitude higher than $k_{\mathrm{B}} T$. The rISC rate is exponentially dependent on $\Delta E_{\mathrm{ST}}$, and it is suggested that a $\Delta E_{\mathrm{ST}}$ of this size is simply too large to enable direct thermally promoted rISC from $\mathrm{T}_{1}$ to $\mathrm{S}_{1}$. With a $\Delta E_{\mathrm{ST}}$ on the order of $0.3 \mathrm{eV}$, there will be little if any relaxation of selection rules to mediate the triplet to singlet spin flip. Conversely, reverse internal conversion by thermal promotion from $T_{1}$ to $T_{n}$ is spin allowed and is hence more probable, where rISC can then occur between $T_{n}$ and $S_{1}$ as these upper states are closer in energy to $S_{1}$ than $T_{1}$. Thermal promotion from $\mathrm{T}_{1}$ to $\mathrm{T}_{n}$ is still an inefficient process that is manifested in the long lifetime observed for HMAT-TRZ emission. In molecules $1 \mathrm{c}$ and $2 \mathrm{c}$, the longer components of the delayed emission are likely not observed because this emission is weaker. We have ruled out TTA emission in HMAT-TRZ by means of power dependence (Figure S2h), and therefore, the rISC between upper-triplet states $\left(\mathrm{T}_{n}\right)$ and the $S_{1}$ state is a more plausible mechanism than vibronically coupled TADF.

Theoretical Investigations. To support our observations, DFT calculations were performed for comparison with experimental data. The ground state geometries of HMATTRZ (Figure S3a) and $\mathbf{1 a}-\mathbf{c}$ and $\mathbf{2 a - c}$ (Figure S3b) were calculated at the rCAM-B3LYP/6-31G(d) level of theory for consistency with the published work on HMAT-TRZ. ${ }^{40}$ The molecular skeleton of HMAT-TRZ is determined by the planar diphenyltriazine electron-accepting moiety and the slightly folded azatriangulene donor $\left(\alpha=2.73^{\circ}\right)$. Such a coplanar D-A molecular orientation leads to significant overlap of HOMO and LUMO wave functions. The deep value of $E_{\text {Hомо }}(-6.21 \mathrm{eV})$ implies the triazine fragment has a strong electron accepting character and is the main reason for the partial frontier orbital decoupling indicated in Figure S3b.

In the case of $\mathbf{1 a}-\mathbf{c}$ and $\mathbf{2 a}-\mathbf{c}$, accepting units with less bulky substituents facilitate torsion around the $\mathrm{D}-\mathrm{A}$ bond. ortho substitution of the phenyl ring of the acceptor in $\mathbf{1 a}-\mathbf{c}$ and $\mathbf{2 a - c}$ leads to an increase in the $\mathrm{D}-\mathrm{A}$ dihedral angle to $43-58^{\circ}$, compared to the biphenyl dihedral angle of $\sim 30^{\circ} .51,52$

Figure 10 suggests a similar electron wave function distribution for $\mathbf{1 a}-\mathbf{c}$ and $\mathbf{2 a - c}$ to HMAT-TRZ. However, there is an increased level of delocalization in $\mathbf{1 a}-\mathbf{c}$ and $\mathbf{2 a - c}$ due to the weaker acceptors and additional symmetry effects. Even stronger delocalization can be observed in the case of $\mathbf{2 a - c}$ and is in agreement with the deeper HOMO values. The calculated deeper $E_{\mathrm{HOMO}}$ values for $\mathbf{2 a}-\mathbf{c}$ are consistent with the trends found by cyclic voltammetry (Table S4).

The theoretical ultraviolet-visible absorption spectrum (Figure S3c) of HMAT-TRZ features two major peaks and extends to $450 \mathrm{~nm}$. The $S_{0} \rightarrow S_{1}$ excitation of HMAT-TRZ has a remarkably high oscillator strength of 0.67 , presumably due to the partial mixing of CT and LE state character observed in the 

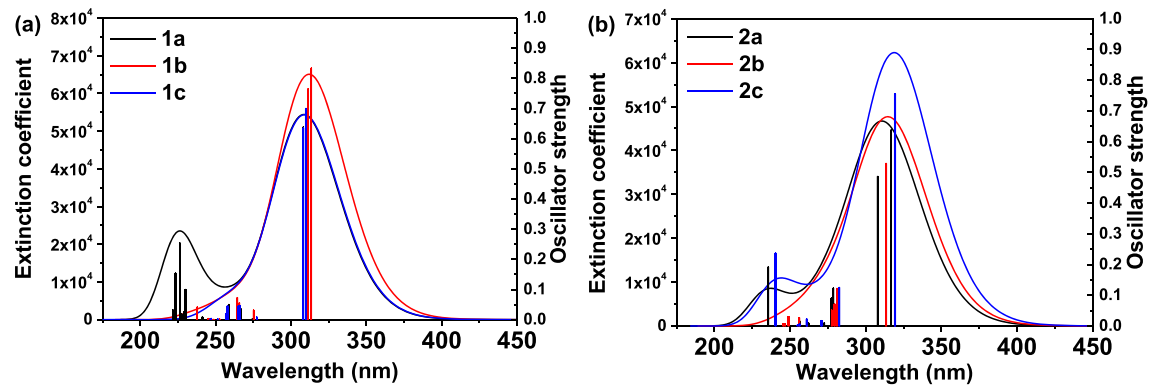

Figure 11. Theoretical ultraviolet-visible spectra of (a) 1a-c and (b) 2a-c [TDA-DFT rCAM-B3LYP/6-31G(d)].

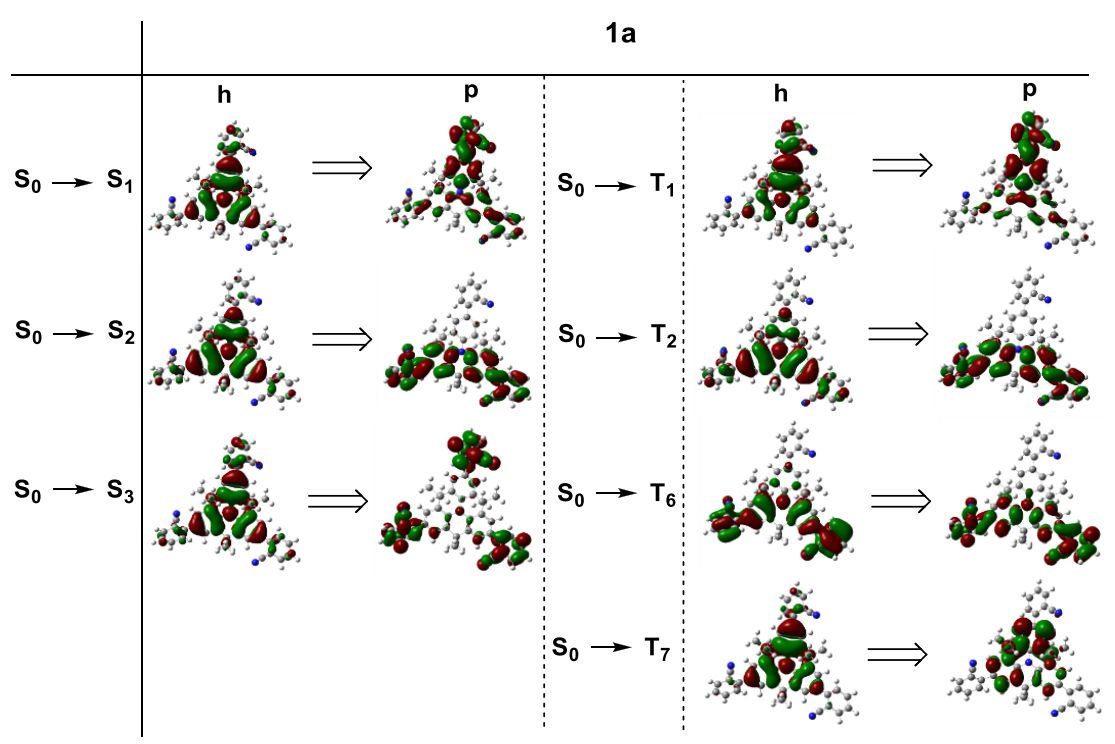

Figure 12. Selected set of singlet and triplet NTOs of 1a [TDA-DFT rCAM-B3LYP/6-31G(d)]. h, hole; p, particle.

experimental spectrum. ${ }^{40}$ The proximity of $\mathrm{T}_{4-6}$ levels to $\mathrm{S}_{1}$ supports the upper-triplet state mechanism of delayed fluorescence (Figure S3d). 32,33,53,54

The calculated absorption spectra of trisubstituted compounds $1 \mathrm{a}-\mathbf{c}$ and $2 \mathrm{a}-\mathrm{c}$ extend to $400-425 \mathrm{~nm}$ (Figure 11). 1a, 2a, and 2c feature two peaks at approximately 225-275 and 315 $\mathrm{nm}$ (only a single low-energy peak was observed for $\mathbf{1 b}, \mathbf{1} \mathbf{c}$, and 2b). 1c exhibited a single low-energy peak of lowest intensity in the experimental absorption spectrum. Unlike in the case of HMAT-TRZ, the intensity of the low-energy peak in $\mathbf{1 a}-\mathbf{c}$ and $\mathbf{2 a}-\mathbf{c}$ is much higher than that of the high-energy peak, with higher $S_{0} \rightarrow S_{1}$ transition oscillator strengths $(\leq 0.9)$. Higher oscillator strengths of the $S_{0} \rightarrow S_{1}$ transition in the case of 1 a-c and $2 \mathbf{a}-\mathbf{c}$ can be assigned to the presence of multiple electronaccepting units and extension of the $\pi$-conjugated system. However, the higher intensity of the low-energy peak is also a signature of the significant LE character of the transitions.

The appearance of several almost degenerate higher triplet states in $\mathbf{1 a}-\mathbf{c}$ and $\mathbf{2 a - c}$ (Figure S3d) can be attributed to higher symmetry. ${ }^{55,56}$ Furthermore, the strong electron acceptors [2,4-bis(trifluoromethyl)pyridine in the case of 1c and isophthalonitrile in the case of $\mathbf{2 c}$ ] narrow the gap between $S_{1}$ and $S_{2}$. Along with the experimentally observed trends in $\Delta E_{\mathrm{ST}}$ $(0.3-0.4 \mathrm{eV})$ for HMAT-TRZ, $\mathbf{1 a}-\mathbf{c}$, and $\mathbf{2 a}-\mathbf{c}$, it is suggested that delayed fluorescence in HMAT-TRZ, $1 \mathrm{c}$, and $2 \mathrm{c}$ molecules all originates from upper-triplet state crossing. Even though the calculated $S_{1}-T_{1}$ gap is overestimated compared to the experimental values (a common feature of DFT), the $S_{1}-T_{n}$ gap (where $n>1$ ) for all calculated molecules is considerably smaller (Figure S3d). Thus, the vicinity of upper-triplet states $\left(\mathrm{T}_{4-8}\right)$ to $\mathrm{S}_{1}$ supports the experimental evidence suggesting an upper-triplet state crossing mechanism. The examples of selected sets of pertinent NTOs for $\mathbf{1 a}$ and $\mathbf{2 a}$ are presented (Figure 12 and Figure S3e). The overlapping but not fully delocalized holes and particles of $S_{0} \rightarrow S_{1,2}$ and $S_{0} \rightarrow T_{1,2}$ in the case of 1a and 2a suggest the partial mixing of the LE and CT character to some degree. Utilization of weaker acceptors with a smaller $\pi$-system in $\mathbf{1 a}-\mathbf{c}$ and $\mathbf{2 a - c}$ compared to the triazine unit in HMAT-TRZ is responsible for the higher degree of wave function delocalization in $\mathbf{1 a}-\mathbf{c}$ and $\mathbf{2 a}-\mathbf{c}$. Consistent with the higher LE character, the level of delocalization is even higher in the case of $2 a-c$.

To assess if stronger electron acceptors have an impact on the optical properties, the relevant NTOs for the compound with the highest degree of CT (1c) are presented (Figure S3f). However, similar trends in delocalization of the electron wave functions are observed. Thus, it is concluded that in the current systems the $\mathrm{D}-\mathrm{A}$ dihedral angle and CT strength play a crucial role in determination of the observed properties.

From all of the spectroscopic data and theoretical calculations presented above, it is suggested that the $\Delta E_{\mathrm{ST}}$ values for all of these materials are too large to support efficient vibronically coupled rISC TADF. If the $\Delta E_{\mathrm{ST}}$ in an observed system is $\geq 0.3$ $\mathrm{eV}$, it is unlikely that any delayed emission will be a consequence of direct rISC from $T_{1}$ to $S_{1}$, and other mechanisms are likely to play a role. In the molecules discussed herein, the $\mathrm{C}-\mathrm{C}$ bridging bond between $\mathrm{D}$ and $\mathrm{A}$ does not effectively electronically decouple the units to minimize $\Delta E_{\mathrm{ST}}$. Effective decoupling of $\mathrm{D}$ 
and $\mathrm{A}$ units is a feature that $\mathrm{C}-\mathrm{N}$ bonded TADF materials have achieved very effectively, but whether they will ever be stable enough in the deep-blue region for commercialization remains in question. The $\mathrm{C}-\mathrm{C}$ bonding provides an interesting avenue for future exploration, but further molecular design is required. However, weak DF is observed in specific solid state hosts, namely, $\mathrm{CBP}$ and $\mathrm{mCBP}$. As the environmental polarity does not control the appearance of DF, it is concluded that the weak DF observed likely comes from an upper-triplet state rISC mechanism via the predominantly local upper-triplet excited states. This helps to rationalize why only CBP-type hosts afford $\mathrm{DF}$, which is likely due to the host-guest packing effect in the films. It is suggested that packing effects exerted by the host perturb the electronic states of the emitters such that an uppertriplet state comes close in energy with $S_{1}$. This $T_{N} / S_{1}$ alignment increases the rate of $T_{N} \rightarrow S_{1}$ crossing such that the thermally activated population of $T_{N}$ by reverse internal conversion $\left(T_{1} \rightarrow\right.$ $\mathrm{T}_{\mathrm{N}}$ ) can lead to rISC that is in competition with internal conversion (IC) back to $T_{1}$. However, the overall rate of delayed fluorescence is rather slow, typically in the millisecond range, not the microsecond lifetime that is commonly observed in vibronically coupled TADF materials. ${ }^{44}$ Thus, the DF in photophysical measurements appears weak because the photogeneration of an initial $T_{1}$ population is low. DFT calculations suggest that HMAT-TRZ has planarized D-A units, both in this work and in the literature. ${ }^{40}$ To summarize, in $\mathrm{C}-\mathrm{C}$ bonded $\mathrm{D}-$ A molecules, a small D-A dihedral angle is unlikely to result in a sufficiently small $\Delta E_{\mathrm{ST}}$ value to produce microsecond-lifetime vibronically coupled TADF, as an increased separation between the $\mathrm{D}$ and $\mathrm{A}$ units is evidently required.

\section{CONCLUSIONS}

Two series of symmetrical molecules have been synthesized with either dimethylmethylene- or diphenylmethylene-functionalized azatriangulene electron donor cores and three pendant electron acceptor units. The donor and acceptor moieties are joined by $\mathrm{C}-\mathrm{C}$ bonds. A detailed photophysical and computational study of these molecules has established that the singlettriplet gap is $>0.3 \mathrm{eV}$ and emission does not occur by a conventional vibronically coupled TADF mechanism. Instead, selected examples (1c and $\mathbf{2 c}$ ) are shown to emit delayed fluorescence via rISC with upper-triplet states. It is proposed that packing effects of the host on the emitters play a key role in whether delayed emission is observed. Obtaining a vibronically coupled TADF molecule with a $\mathrm{C}-\mathrm{C}$ bonded $\mathrm{D}-\mathrm{A}$ bridge therefore remains a significant challenge. It is apparent that significantly larger $\mathrm{D}-\mathrm{A}$ dihedral angles, compared to those in the present molecules, are required to effectively separate the HOMO and LUMO. This molecular design is an interesting and promising future avenue for producing molecules with high materials stability and vibronically coupled TADF. This work also shows how planarized donor-acceptor structures (e.g., HMAT-TRZ) are unlikely to yield conventional vibronically coupled TADF due to excessive electronic communication between the donor and acceptor. However, planarization of DA systems is a promising design for achieving delayed fluorescence via upper-triplet state crossing. The new central donor core unit in $\mathbf{2 a - c}$ results in considerably improved thermal stability compared to that of $1 \mathbf{a}-\mathbf{c}$. Through rational molecular design, it is now also possible to control whether the oxidation processes are reversible in these $\mathrm{D}-\mathrm{A}_{3}$ systems.

This work has also highlighted that it is essential to rigorously characterize the photophysical properties of new molecules.
Clear details of delays and integration times of emission components are essential, to separate emission features and clearly understand which types of triplet-harvesting processes are occurring. Molecules emitting via different mechanisms could significantly affect relevant optoelectronic properties due to variation in excited state lifetimes. Molecules emitting via a vibronic-coupling mechanism or an upper-triplet state crossing mechanism on first inspection can show highly similar photophysics; however, subtle differences are apparent and have been distinguished in this series of molecules as a result of systematic molecular design and detailed photophysical analysis.

\section{ASSOCIATED CONTENT}

\section{S Supporting Information}

The Supporting Information is available free of charge on the ACS Publications website at DOI: 10.1021/acs.chemmater.9b01184.

${ }^{1} \mathrm{H}$ and ${ }^{13} \mathrm{C}\left\{{ }^{1} \mathrm{H}\right\}$ NMR spectra, absorption spectra, CV traces and data, additional emission data, and computational data (PDF)

CIFs for Cambridge Structural Database entries 1902251-1902257 (ZIP)

\section{AUTHOR INFORMATION}

\section{Corresponding Authors}

*E-mail: m.r.bryce@durham.ac.uk.

*E-mail: a.p.monkman@durham.ac.uk.

ORCID

Jonathan S. Ward: 0000-0002-4985-8572

Andrei S. Batsanov: 0000-0002-4912-0981

Andrew P. Monkman: 0000-0002-0784-8640

Martin R. Bryce: 0000-0003-2097-7823

\section{Author Contributions}

J.S.W., N.A.K., P.L.d.S., and D.G.C. all contributed equally to the experimental work. J.S.W. led the writing and coordination of the manuscript with assistance from all authors. J.S.W. and D.G.C. designed, synthesized, and characterized the molecules under the supervision of M.R.B. D.G.C. performed the majority of CV measurements with assistance from J.S.W. P.L.d.S. and N.A.K. performed the photophysical measurements under the supervision of A.P.M. N.A.K. performed and reported all of the DFT calculations. J.S.W. and D.G.C. grew crystals suitable for Xray crystallography, which was performed and reported by A.S.B.

\section{Notes}

The authors declare no competing financial interest.

\section{ACKNOWLEDGMENTS}

Dr. Iain Wright (Loughborough University, Loughborough, U.K.) is acknowledged for discussion during interpretation of the electrochemical data. The authors thank EPRSC for Grant EP/L02621X/1. J.S.W., N.A.K., M.R.B., and A.P.M. are thankful for EU Horizon 2020 Grant 732103 (HyperOLED).

\section{REFERENCES}

(1) Li, S. W.; Yu, C. H.; Ko, C. L.; Chatterjee, T.; Hung, W. Y.; Wong, K. T. Cyanopyrimidine-Carbazole Hybrid Host Materials for HighEfficiency and Low-Efficiency Roll-Off TADF OLEDs. ACS Appl. Mater. Interfaces 2018, 10, 12930-12936.

(2) Zhang, D.; Cao, X. D.; Wu, Q. J.; Zhang, M. C.; Sun, N.; Zhang, X. P.; Tao, Y. T. Purely organic materials for extremely simple all-TADF white OLEDs: a new carbazole/oxadiazole hybrid material as a dual- 
role non-doped light blue emitter and highly efficient orange host. J. Mater. Chem. C 2018, 6, 3675-3682.

(3) Zhang, D. D.; Cai, M. H.; Zhang, Y. G.; Zhang, D. Q.; Duan, L. Highly Efficient Simplified Single-Emitting-Layer Hybrid WOLEDs with Low Roll-off and Good Color Stability through Enhanced Forster Energy Transfer. ACS Appl. Mater. Interfaces 2015, 7, 28693-28700.

(4) Yang, H.; Liang, Q.; Han, C.; Zhang, J.; Xu, H. A Phosphanthrene Oxide Host with Close Sphere Packing for Ultralow-Voltage-Driven Efficient Blue Thermally Activated Delayed Fluorescence Diodes. Adv. Mater. 2017, 29, 1700553.

(5) Ihn, S. G.; Lee, N.; Jeon, S. O.; Sim, M.; Kang, H.; Jung, Y.; Huh, D. H.; Son, Y. M.; Lee, S. Y.; Numata, M.; Miyazaki, H.; GomezBombarelli, R.; Aguilera-Iparraguirre, J.; Hirzel, T.; Aspuru-Guzik, A.; Kim, S.; Lee, S. An Alternative Host Material for Long-Lifespan Blue Organic Light-Emitting Diodes Using Thermally Activated Delayed Fluorescence. Adv. Sci. 2017, 4, 1600502.

(6) Fang, Z.; Chellappan, V.; Webster, R. D.; Ke, L.; Zhang, T. F.; Liu, B.; Lai, Y. H. Bridged-triarylamine starburst oligomers as hole transporting materials for electroluminescent devices. J. Mater. Chem. 2012, 22, 15397-15404.

(7) Xiong, Y.; Wu, B.; Zheng, X. Y.; Zhao, Z.; Deng, P.; Lin, M.; Tang, B. Z.; Ong, B. S. Novel Dimethylmethylene-Bridged TriphenylaminePDI Acceptor for Bulk-Heterojunction Organic Solar Cells. Adv. Sci. 2017, 4, 1700110.

(8) Jin, E. Q.; Du, C.; Wang, M.; Li, W. W.; Li, C. H.; Wei, H. D.; Bo, Z. S. Dibenzothiophene-Based Planar Conjugated Polymers for High Efficiency Polymer Solar Cells. Macromolecules 2012, 45, 7843-7854.

(9) Echeverry, C. A.; Cotta, R.; Insuasty, A.; Ortiz, A.; Martin, N.; Echegoyen, L.; Insuasty, B. Synthesis of novel light harvesters based on perylene imides linked to triphenylamines for Dyes Sensitized Solar Cells. Dyes Pigm. 2018, 153, 182-188.

(10) Boschloo, G.; Hagfeldt, A. Photoinduced absorption spectroscopy as a tool in the study of dye-sensitized solar cells. Inorg. Chim. Acta 2008, 361, 729-734.

(11) Knopfmacher, O.; Hammock, M. L.; Appleton, A. L.; Schwartz, G.; Mei, J.; Lei, T.; Pei, J.; Bao, Z. Highly stable organic polymer fieldeffect transistor sensor for selective detection in the marine environment. Nat. Commun. 2014, 5, 2954.

(12) Wang, Y.; Liu, D. D.; Ikeda, S.; Kumashiro, R.; Nouch, R.; Xu, Y. X.; Shang, H.; Ma, Y. G.; Tanigaki, K. Ambipolar behavior of 2,5diphenyl-1,4-distyrylbenzene based field effect transistors: An experimental and theoretical study. Appl. Phys. Lett. 2010, 97, No. 033305.

(13) Zhou, Y. P.; Xie, Q. J. Hyaluronic acid-coated magnetic nanoparticles-based selective collection and detection of leukemia cells with quartz crystal microbalance. Sens. Actuators, B 2016, 223, 9-14.

(14) Steinegger, A.; Klimant, I.; Borisov, S. M. Purely Organic Dyes with Thermally Activated Delayed FluorescenceA Versatile Class of Indicators for Optical Temperature Sensing. Adv. Opt. Mater. 2017, 5, 1700372.

(15) Li, X. C.; Wang, C. Y.; Wan, Y.; Lai, W. Y.; Zhao, L.; Yin, M. F.; Huang, W. A T-shaped triazatruxene probe for the naked-eye detection of $\mathrm{HCl}$ gas with high sensitivity and selectivity. Chem. Commun. 2016, $52,2748-2751$.

(16) Schmidt, C. E.; Shastri, V. R.; Vacanti, J. P.; Langer, R. Stimulation of neurite outgrowth using an electrically conducting polymer. Proc. Natl. Acad. Sci. U. S. A. 1997, 94, 8948-8953.

(17) Simon, D. T.; Kurup, S.; Larsson, K. C.; Hori, R.; Tybrandt, K.; Goiny, M.; Jager, E. W.; Berggren, M.; Canlon, B.; Richter-Dahlfors, A. Organic electronics for precise delivery of neurotransmitters to modulate mammalian sensory function. Nat. Mater. 2009, 8, 742-746.

(18) Lin, T. A.; Chatterjee, T.; Tsai, W. L.; Lee, W. K.; Wu, M. J.; Jiao, M.; Pan, K. C.; Yi, C. L.; Chung, C. L.; Wong, K. T.; Wu, C. C. Sky-Blue Organic Light Emitting Diode with 37\% External Quantum Efficiency Using Thermally Activated Delayed Fluorescence from SpiroacridineTriazine Hybrid. Adv. Mater. 2016, 28, 6976-6983.

(19) dos Santos, P. L.; Ward, J. S.; Congrave, D. G.; Batsanov, A. S.; Eng, J.; Stacey, J. E.; Penfold, T. J.; Monkman, A. P.; Bryce, M. R. Triazatruxene: A Rigid Central Donor Unit for a $\mathrm{D}-\mathrm{A}_{3}$ Thermally Activated Delayed Fluorescence Material Exhibiting Sub-Microsecond
Reverse Intersystem Crossing and Unity Quantum Yield via Multiple Singlet-Triplet State Pairs. Adv. Sci. 2018, 5, 1700989.

(20) Miao, Y.; Tao, P.; Wang, K.; Li, H.; Zhao, B.; Gao, L.; Wang, H.; $\mathrm{Xu}, \mathrm{B}$.; Zhao, Q. Highly Efficient Red and White Organic LightEmitting Diodes with External Quantum Efficiency beyond 20\% by Employing Pyridylimidazole-Based Metallophosphors. ACS Appl. Mater. Interfaces 2017, 9, 37873-37882.

(21) Yang, X.; Guo, H.; Liu, B.; Zhao, J.; Zhou, G.; Wu, Z.; Wong, W.Y. Diarylboron-Based Asymmetric Red-Emitting Ir(III) Complex for Solution-Processed Phosphorescent Organic Light-Emitting Diode with External Quantum Efficiency above 28\%. Adv. Sci. 2018, 5, 1701067.

(22) Li, G. F.; Congrave, D. G.; Zhu, D. X.; Su, Z. M.; Bryce, M. R. Recent advances in luminescent dinuclear iridium(III) complexes and their application in organic electroluminescent devices. Polyhedron 2018, 140, 146-157.

(23) Tuong Ly, K.; Chen-Cheng, R.-W.; Lin, H.-W.; Shiau, Y.-J.; Liu, S.-H.; Chou, P.-T.; Tsao, C.-S.; Huang, Y.-C.; Chi, Y. Near-infrared organic light-emitting diodes with very high external quantum efficiency and radiance. Nat. Photonics 2017, 11, 63-68.

(24) Fleetham, T.; Li, G.; Wen, L.; Li, J. Efficient "Pure" Blue OLEDs Employing Tetradentate Pt Complexes with a Narrow Spectral Bandwidth. Adv. Mater. 2014, 26, 7116-7121.

(25) Cebrian, C.; Mauro, M. Recent advances in phosphorescent platinum complexes for organic light-emitting diodes. Beilstein J. Org. Chem. 2018, 14, 1459-1481.

(26) Uoyama, H.; Goushi, K.; Shizu, K.; Nomura, H.; Adachi, C. Highly efficient organic light-emitting diodes from delayed fluorescence. Nature 2012, 492, 234-238.

(27) Wilkinson, F.; Horrocks, A. R. Luminescence in Chemistry; Bowen, E. J., Ed.; Van Nostrand: London, 1968.

(28) Gibson, J.; Penfold, T. J. Nonadiabatic coupling reduces the activation energy in thermally activated delayed fluorescence. Phys. Chem. Chem. Phys. 2017, 19, 8428-8434.

(29) Etherington, M. K.; Gibson, J.; Higginbotham, H. F.; Penfold, T. J.; Monkman, A. P. Revealing the spin-vibronic coupling mechanism of thermally activated delayed fluorescence. Nat. Commun. 2016, 7, 13680.

(30) Gibson, J.; Monkman, A. P.; Penfold, T. J. The Importance of Vibronic Coupling for Efficient Reverse Intersystem Crossing in Thermally Activated Delayed Fluorescence Molecules. ChemPhysChem 2016, 17, 2956-2961.

(31) Penfold, T. J.; Dias, F. B.; Monkman, A. P. The theory of thermally activated delayed fluorescence for organic light emitting diodes. Chem. Commun. 2018, 54, 3926-3935.

(32) Northey, T.; Eng, J.; Keane, T.; Penfold, T. J. Understanding the Potential for Efficient Triplet Harvesting with Hot Excitons. Faraday Discuss. 2019, DOI: 10.1039/C8FD00174J.

(33) Huang, R. J.; Avo, J.; Northey, T.; Chaning-Pearce, E.; dos Santos, P. L.; Ward, J. S.; Data, P.; Etherington, M. K.; Fox, M. A.; Penfold, T. J.; Berberan-Santos, M. N.; Lima, J. C.; Bryce, M. R.; Dias, F. $B$. The contributions of molecular vibrations and higher triplet levels to the intersystem crossing mechanism in metal-free organic emitters. J. Mater. Chem. C 2017, 5, 6269-6280.

(34) Parker, C. A.; Hatchard, C. G. Triplet-Singlet Emission in Fluid Solutions - Phosphorescence of Eosin. Trans. Faraday Soc. 1961, 57, 1894-1904.

(35) Cai, X. Y.; Su, S. J. Marching Toward Highly Efficient, Pure-Blue, and Stable Thermally Activated Delayed Fluorescent Organic LightEmitting Diodes. Adv. Funct. Mater. 2018, 28, 1802558.

(36) Song, W.; Lee, J. Y. Degradation Mechanism and Lifetime Improvement Strategy for Blue Phosphorescent Organic LightEmitting Diodes. Adv. Opt. Mater. 2017, 5, 1600901.

(37) Cao, X. D.; Zhang, D.; Zhang, S. M.; Tao, Y. T.; Huang, W. CNContaining donor-acceptor-type small-molecule materials for thermally activated delayed fluorescence OLEDs. J. Mater. Chem. C 2017, 5, 7699-7714. 
(38) Amara, J. P.; Swager, T. M. Conjugated polymers with geminal trifluoromethyl substituents derived from hexafluoroacetone. Macromolecules 2006, 39, 5753-5759.

(39) Jiang, Z. Q.; Chen, Y. H.; Yang, C. L.; Cao, Y.; Tao, Y. T.; Qin, J. G.; Ma, D. G. A Fully Diarylmethylene-Bridged Triphenylamine Derivative as Novel Host for Highly Efficient Green Phosphorescent OLEDs. Org. Lett. 2009, 11, 1503-1506.

(40) Chen, X. K.; Tsuchiya, Y.; Ishikawa, Y.; Zhong, C.; Adachi, C.; Bredas, J. L. A New Design Strategy for Efficient Thermally Activated Delayed Fluorescence Organic Emitters: From Twisted to Planar Structures. Adv. Mater. 2017, 29, 1702767.

(41) Ward, J. S.; Nobuyasu, R. S.; Fox, M. A.; Batsanov, A. S.; Santos, J.; Dias, F. B.; Bryce, M. R. Bond rotations and heteroatom effects in donor-acceptor-donor molecules: Implications for thermally activated delayed fluorescence and room temperature phosphorescence. J. Org. Chem. 2018, 83, 14431-14442.

(42) dos Santos, P. L.; Ward, J. S.; Bryce, M. R.; Monkman, A. P. Using Guest-Host Interactions To Optimize the Efficiency of TADF OLEDs. J. Phys. Chem. Lett. 2016, 7, 3341-3346.

(43) dos Santos, P. L.; Ward, J. S.; Data, P.; Batsanov, A. S.; Bryce, M. R.; Dias, F. B.; Monkman, A. P. Engineering the singlet-triplet energy splitting in a TADF molecule. J. Mater. Chem. C 2016, 4, 3815-3824.

(44) Hosokai, T.; Matsuzaki, H.; Nakanotani, H.; Tokumaru, K.; Tsutsui, T.; Furube, A.; Nasu, K.; Nomura, H.; Yahiro, M.; Adachi, C. Evidence and mechanism of efficient thermally activated delayed fluorescence promoted by delocalized excited states. Sci. Adv. 2017, 3, e1603282.

(45) Tanaka, H.; Shizu, K.; Miyazaki, H.; Adachi, C. Efficient green thermally activated delayed fluorescence (TADF) from a phenoxazinetriphenyltriazine (PXZ-TRZ) derivative. Chem. Commun. 2012, 48, 11392-11394.

(46) Cha, J.-R.; Lee, C. W.; Lee, J. Y.; Gong, M.-S. Design of ortholinkage carbazole-triazine structure for high-efficiency blue thermally activated delayed fluorescent emitters. Dyes Pigm. 2016, 134, 562-568.

(47) Zhang, D. D.; Cai, M. H.; Zhang, Y. G.; Zhang, D. Q.; Duan, L. Sterically shielded blue thermally activated delayed fluorescence emitters with improved efficiency and stability. Mater. Horiz. 2016, 3, 145-151.

(48) Jeon, S. O.; Jang, S. E.; Son, H. S.; Lee, J. Y. External quantum efficiency above $20 \%$ in deep blue phosphorescent organic lightemitting diodes. Adv. Mater. 2011, 23, 1436-1441.

(49) Lee, S. J.; Seo, J. H.; Kim, G. Y.; Kim, Y. K. A Study on the Phosphorescent Blue Organic Light-Emitting Diodes Using Various Host Materials. Mol. Cryst. Liq. Cryst. 2009, 507, 345-352.

(50) Xie, G. Z.; Chen, D. J.; Li, X. L.; Cai, X. Y.; Li, Y. C.; Chen, D. C.; Liu, K. K.; Zhang, Q.; Cao, Y.; Su, S. J. Polarity-Tunable Host Materials and Their Applications in Thermally Activated Delayed Fluorescence Organic Light-Emitting Diodes. ACS Appl. Mater. Interfaces 2016, 8, 27920-27930.

(51) Eaton, V. J.; Steele, D. Dihedral Angle of Biphenyl in Solution and Molecular Force-Field. J. Chem. Soc., Faraday Trans. 2 1973, 69, 1601-1608.

(52) Grein, F. Twist angles and rotational energy barriers of biphenyl and substituted biphenyls. J. Phys. Chem. A 2002, 106, 3823-3827.

(53) Lee, J.; Jeong, C.; Batagoda, T.; Coburn, C.; Thompson, M. E.; Forrest, S. R. Hot excited state management for long-lived blue phosphorescent organic light-emitting diodes. Nat. Commun. 2017, 8, 15566.

(54) Jankus, V.; Aydemir, M.; Dias, F. B.; Monkman, A. P. Generating Light from Upper Excited Triplet States: A Contribution to the Indirect Singlet Yield of a Polymer OLED, Helping to Exceed the 25\% Singlet Exciton Limit. Adv. Sci. 2016, 3, 1500221.

(55) Detert, H.; Lehmann, M.; Meier, H. Star-Shaped Conjugated Systems. Materials 2010, 3, 3218-3330.

(56) Sato, T.; Uejima, M.; Tanaka, K.; Kaji, H.; Adachi, C. A lightemitting mechanism for organic light-emitting diodes: molecular design for inverted singlet-triplet structure and symmetry-controlled thermally activated delayed fluorescence. J. Mater. Chem. C 2015, 3, 870-878. 